\title{
Wissensmanagement
}

\author{
Sophie März*, DongBack Seo und Christa Womser-Hacker
}

\section{Wie beeinflusst die Generationszugehörigkeit das Teilen von Wissen in Unternehmen?}

\author{
Eine empirische Studie
}

https://doi.org/10.1515/iwp-2020-2103

Zusammenfassung: Das Teilen von Wissen ist für die heutige Informationsgesellschaft eine der wichtigsten Herausforderungen. Um ein erfolgreiches und innovatives Agieren zum Beispiel in Unternehmen zu ermöglichen, ist eine kontinuierliche Strategie im Umgang mit Wissen und Information unerlässlich. Diese ist abhängig von gutem Wissensmanagement und vor allem von der Bereitschaft, Wissen zu teilen. Da Altersdiversität und damit einhergehende Generationenkonflikte den unternehmerischen Alltag stark prägen, wird in dieser Arbeit auf die Unterschiede zwischen jüngeren und älteren Arbeitnehmern ${ }^{1}$ eingegangen. Wie können verschiedene Altersgruppen motiviert werden, ihr Wissen zu teilen? Welche Umstände sollten vermieden werden, damit keine Hürden entstehen? Um diesen Fragen nachzugehen, wurde eine Umfrage in deutschen Unternehmen mit einer Rücklaufquote von 236 Personen durchgeführt. Diese veranschaulicht, dass ältere Mitarbeiter mehr dazu tendieren, ihr Wissen zu teilen als ihre jüngeren Kollegen. Vor allem die Befürchtung, sich zu blamieren und Fehler zu machen, scheint der jüngeren Generation Probleme zu bereiten. Für Unternehmen ist es wichtig, Unterschiede und Gemeinsamkeiten zu erkennen, um Strategien und Instrumentarien zu entwickeln, die eine gelungene Interaktion verschiedener Altersgruppen ermöglichen.

1 Es wird zur besseren Lesbarkeit das generische Maskulinum verwendet. Gemeint sind aber alle Geschlechter.

\footnotetext{
*Kontaktperson: Sophie März, Universität Hildesheim, Institut für Informationswissenschaft und Sprachtechnologie, Universitätsplatz 1, 31141 Hildesheim, E-Mail: maerz@uni-hildesheim.de Prof. DongBack Seo, Ph. D., Chungbuk National University, Department of Management Information Systems, 567 Baekje-daero, Deokjin-gu, Jeonju-si, Jeollabuk-do, 54896 Republic of Korea, E-Mail: dseo@chungbuk.ac.kr

Prof. Dr. Christa Womser-Hacker, Universität Hildesheim, Institut für Informationswissenschaft und Sprachtechnologie, Universitätsplatz 1, 31141 Hildesheim, E-Mail: womser@uni-hildesheim.de
}

Deskriptoren: Wissensmanagement, Wissensteilung, Wissenslücke, Generationenkonflikt, Arbeitsplatz, Altersdiversität, Informationsgesellschaft, Motivationstheorie, Zusammenarbeit, Individualismus, Kollektivismus, Altersstereotype, demographischer Wandel, Renteneintritt der Baby Boomer

\section{How does generational affiliation influence the sharing of knowledge in companies?}

An empirical study

Abstract: Knowledge sharing is an important challenge in today's information society. In order to enable organizations to operate successfully and innovatively, continuing working and developing is essential to them. This progress depends on good knowledge management and above all on the starting point: knowledge sharing. Due to the fact that age diversity, generational gaps and associated conflicts strongly influence the daily business life, this paper investigates differences between younger and older employees in sharing their knowledge. How can different age groups be motivated? Which circumstances should be avoided to prevent barriers? In order to find out about the various factors, an online survey was conducted in German organizations with a response rate of 236 persons. The results show that more aged employees tend to share their knowledge more likely than their younger colleagues. Especially the fear of losing their face which contains the fear of being embarrassed and making mistakes seems to cause problems for younger people. It is important for organizations to identify differences and similarities in order to develop strategies and tools to enable successful interaction between different age groups.

Descriptors: Knowledge management, Knowledge sharing, Knowledge gap, Generation gap, Workplace, age-diversity, Information society, Motivation theory, Cooperation, Individualism, Collectivism, Ageing-stereotypes, Demographic change, Retirement of baby boomers 
Comment l'appartenance à une génération influence-t-elle le partage des connaissances dans les entreprises ? Une étude empirique

Résumé: le partage des connaissances est l'un des défis les plus importants de la société de l'information actuelle. Pour permettre une action réussie et innovante, par exemple dans les entreprises, il est essentiel de disposer d'une stratégie continue en matière de connaissances et d'informations. Cela dépend d'une bonne gestion des connaissances et, surtout, de la volonté de partager les connaissances. Étant donné que la diversité des âges et les conflits générationnels qui y sont associés influencent fortement la vie quotidienne des entreprises, cet article se concentrera sur les différences entre les jeunes et les plus âgés. Comment motiver les différents groupes d'âge à partager leurs connaissances? Quelles sont les circonstances à éviter pour éviter les obstacles ? Pour répondre à ces questions, une enquête a été menée dans des entreprises allemandes, avec un taux de réponse de 236 personnes. Les résultats montrent que les employés plus âgés ont davantage tendance à partager leurs connaissances que leurs jeunes collègues. Par-dessus tout, la peur de perdre la face et de faire des erreurs semble poser des problèmes à la jeune génération. Il est important pour les entreprises d'identifier les différences et les similitudes afin de développer des stratégies et des outils qui permettent une interaction réussie entre les différents groupes d'âge.

Descripteurs: Gestion des connaissances, Partage des connaissances, Fossé des connaissances, Conflit des générations, Lieu de travail, Diversité des âges, Société de l'information, Théorie de la motivation, Coopération, Individualisme, Collectivisme, Stéréotypes liés à l'âge, Changement démographique, Retraite des baby-boomers

\section{Wissensteilung am altersdiversen Arbeitsplatz}

Für Organisationen stellt es eine große Herausforderung dar, gute Strategien für einen aktiven Umgang mit Information und Wissen zu entwickeln. Das Teilen, der Austausch und die Erweiterung von Wissen sind dabei von zentraler Bedeutung, um konkurrenzfähig, innovativ und leistungsfähig zu bleiben (Reychav \& Weisberg, 2010, S. 287; Razak, Pangil, Zin, Yunus \& Asnawi, 2016, S. 546; Farooq, 2018). Nur durch Kommunikation und Zusammenschluss erarbeiteten Wissens kann eine aktuelle Wissensbasis als Grundlage für eine Weiterentwicklung herangezogen werden (Nonaka, 1994, S. 14; Bock, Zmund,
Kim \& Lee, 2005, S. 89). Dieses Wissen innerhalb von Organisationen, das auf dem individuellen Wissen der einzelnen Mitarbeiter beruht, ist ein wichtiges immaterielles Gut (Widaja, 2019, S. 89).

Im Laufe der nächsten Jahre werden immer mehr Arbeitnehmer der Baby Boomer Generation in den Ruhestand gehen. Dadurch sehen sich die Unternehmen der Gefahr von Wissenseinbußen ausgesetzt, wenn erarbeitetes Wissen nicht an jüngere Mitarbeiter weitergegeben oder anderweitig dokumentiert wird (DeLong, 2004; Slagter, 2007, S. 83; Burmeister \& Deller, 2016).

Auch der demographische Wandel und die alternde Gesellschaft stellen für Organisationen Herausforderungen dar (Germany trade \& invest [GTAI], 2020; Eurostat, 2019). Diversität in Bezug auf Alter und Erfahrung steigt innerhalb der Unternehmen an (Rudolph \& Zacher, 2015). Mehr Kommunikation und Austausch verschiedener Generationen sind gefordert (Kunze, Boehm \& Bruch, 2011, S. 268; McGuire, Todnem \& Hutchings, 2007, S. 529).

Altersdiversität am Arbeitsplatz kann verschiedene Auswirkungen haben und das Verhalten der Beteiligten beeinflussen. So steigt die Möglichkeit von Konflikten ( $\mathrm{Ru}-$ dolph \& Zacher, 2015), Desinteresse an Weiterbildung (Lagacé, Charmarkeh, Zaky \& Firzly, 2016), der Intention, das Unternehmen zu verlassen (Iweins, Desmette, Yzerbyt \& Stinglhamber, 2013), unterschiedlicher Kommunikationsmuster zwischen den Gesprächsteilnehmern (Wok \& Hashim, 2013) und negativer Emotionen und Einstellungen zum Arbeitsplatz (Kunze \& Menges, 2017).

Es ist anzunehmen, dass die verschiedenen Generationen unterschiedliche Beweggründe haben, ihr Wissen mit Kollegen (nicht) zu teilen. Was motiviert Arbeitnehmer verschiedener Altersgruppen, ihr Wissen zu teilen und was hindert sie daran? Worin bestehen die Unterschiede zwischen jüngeren und älteren Personen in ihrer Motivation und den wahrgenommenen Hürden? Hierzu beantworteten 236 Teilnehmer deutscher Unternehmen einen Online-Fragebogen, der verschiedene Faktoren wie Vertrauen, Teamarbeit, extrinsische Anreize und viele weitere Komponenten beinhaltet.

Vor der Darstellung der Ergebnisse und der ausgearbeiteten Praxisempfehlungen werden der aktuelle State of the Art, die Methode und das zugrundeliegende Forschungsmodell erklärt. 


\section{Stand der Forschung: Generationen und das Teilen von Wissen}

Im Bereich des generationenbasierten Teilens von Wissen liegen nur wenige Studien vor, die die zwei großen Forschungsgebiete des Generationenkonfliktes und der Wissensteilung kombinieren. Bestehende Publikationen sind methodisch meist qualitativer Natur und stammen aus verschiedenen Disziplinen wie der Betriebswirtschaftslehre, Psychologie oder der Informationswissenschaft. Dies zeigt zum einen die Bandbreite und Bedeutung in den unterschiedlichen Bereichen und zum anderen die Notwendigkeit, das Thema aus verschiedenen Perspektiven zu betrachten.

Wissensmanagement wird in unterschiedlichen Forschungskontexten unterschiedlich definiert (Girard \& Girard, 2015). Nach Anand und Singh (2011) ist Wissensmanagement „[...] the explicit and systematic management of vital knowledge and its associated processes of creating, gathering, organizing, diffusion, use and exploitation” (S. 932). Demnach bezieht sich das Wissenskonzept auf verschiedene Prozesse innerhalb eines Unternehmens. Das Wissen wird kreiert, organisiert, genutzt und verwertet (Anand \& Sing, 2011, S. 932). Voraussetzung ist das Vorhandensein von Wissen. Dieses muss anschließend geteilt werden, was die Kernaktivität für jedes weitere Vorgehen darstellt. Kankanhalli und Tan (2005) definieren die Wissensteilung als „[...]the core and the most difficult activity of knowledge management” (S. 113). Es handelt sich hierbei um einen individuellen und freiwilligen Prozess, der durch Emotionen, Einstellungen und die Umgebung beeinflusst wird (Bock et al., 2005, S. 89; Krok, 2013, S. 102; Pee \& Lee, 2015, S. 4). Somit müssen Faktoren erkannt werden, die die betreffenden Personen motivieren oder daran hindern, ihr Wissen zu teilen. Das Zusammentreffen verschiedener Generationen am Arbeitsplatz kann $u$. a. beteiligt sein.

Auch das Konzept einer Generation ist nicht klar definiert. Es bestehen verschiedene Ansätze und Verwendungen in der Literatur. Eine weit verbreitete soziologische Sichtweise ist die Einteilung von Personen nach verschiedenen Kategorien, abhängig von ihrem Geburtsjahr: Baby Boomer (1956-1965), Generation X (1966-1980), Generation Y (1981-1995), Generation Z (1996- ) (Klaffke \& Schwarzenbart, 2013). Eine andere Auffassung bezieht sich auf die charakteristischen Eigenschaften von Personen unterschiedlichen Alters. Demnach befinden sich Menschen im gleichen Alter in einer ähnlichen Situation und durchlebten ähnliche Erfahrungen, die sie von Per- sonen anderen Alters unterscheiden. Hierzu zählen unter anderem historische Ereignisse wie die Mondlandung oder 9/11 (Constanza, Badger, Fraser, Severt \& Gade, 2012, S. 367).

Des Weiteren wird versucht, das Konzept Generation durch die soziale Identität zu definieren. Hiernach sind verschiedene soziale und persönliche Komponenten involviert. Soziale Komponenten sind Charakteristika wie Geschlecht, Nationalität und Alter, persönliche Komponenten beziehen sich auf Glaubens- und Wertvorstellungen (Ely, 1994, S. 205; McGuire et al., 2007, S. 569). Kuyken (2012) ist der Meinung, dass es selbst in definierten Generationen unterschiedliche Wissensgemeinschaften gibt. Beispielsweise können eine 40-jährige Krankenschwester und eine 40-jährige IT-Kauffrau in ihren Vorstellungen und Fähigkeiten stark voneinander abweichen und über unterschiedliches Wissen verfügen (Kuyken, 2012, S. 276). Eine andere Perspektive impliziert die biologische Definition. Hierbei wird durch die Geburt von Nachwuchs die nächste Generation eingeleitet, die sich fortpflanzt und weitere Generationen entstehen lässt. Dies zeigt sich zum Beispiel im Generationenwechsel bei Familienunternehmen (Woodfield \& Husted, 2017).

Diese Arbeit beschäftigt sich mit Generationen am Arbeitsplatz, weshalb explizit auf Unterschiede zwischen älteren und jüngeren Personen geachtet wird. Doch welche Eigenschaften, Charakteristika und Fähigkeiten unterscheiden Personen anhand ihres Alters von anderen?

Verschiedene Studien zum Thema Generationen am Arbeitsplatz ohne Bezug auf das Teilen von Wissen haben bereits unterschiedliche Eigenschaften, Einstellungen und Fähigkeiten von Arbeitnehmern verschiedenen Alters ausfindig gemacht. Aus Sicht dieser Untersuchungen sehen sich ältere Kollegen eher einer Generation zugehörig, als es jüngere tun (Lyons \& Schweitzer, 2016). Jüngere Personen empfinden vor allem Status und Freiheit sehr wichtig, wohingegen ältere Kollegen Arbeitswerte und die Bezahlung als bedeutend erachten (Cennamo \& Gardner, 2008).

Bei einer Befragung von 575 malaysischen Arbeitnehmern konnte ein unterschiedliches Kommunikationsverhalten zwischen älteren und jüngeren Personen festgestellt werden (Wok \& Hashim, 2013). Auch die bevorzugte Art des Arbeitsplatzes unterscheidet sich, wohingegen alle Beteiligten Mentoring und teambasierte Arbeitsumgebungen als angenehm empfinden (Joy \& Haynes, 2011). Besteht ein guter Umgang zwischen den verschiedenen Generationen am Arbeitsplatz, sinkt die Intention der Arbeitnehmer, das Unternehmen zu verlassen (Iweins et al., 2013). Ist dies jedoch nicht der Fall und es kommt zu Altersstereotypen, welche altersbedingte Vor- 
behalte in Bezug auf Verhalten und Fähigkeiten implizieren (Peters et al., 2019), können negative Auswirkungen für Organisationen entstehen. Vorbehalte gegenüber älteren oder jüngeren Arbeitnehmern schädigen das Arbeitsklima und die Wahrnehmung von Kollegen (Lagacé et al., 2016). Des Weiteren sind ein negativer Einfluss auf die Leistung der Mitarbeiter und ein sinkendes psychologisches Wohlbefinden möglich (von Hippel, Kalokerinos \& Henry, 2013). Nach Rahman, Daud und Hassan (2017) können Unterschiede der Generationen auch das Verhalten beim Teilen von Wissen beeinflussen. Tritt hier ein negativer Effekt im Austausch von Wissen ein, kann die Teamleistung sinken (Ellwart, Bündgens \& Rack, 2013).

Das Teilen von Wissen mit dem entsprechenden Kommunikationsaspekt und die Teamarbeit hängen somit zusammen. Aus diesem Grund ist es essentiell zu erforschen, welche Auswirkung eine gute Teamarbeit für Personen unterschiedlichen Alters hat, Wissen zu teilen (Iweins et al. 2013). Unterschiede zwischen Generationen müssen verstanden und erkannt werden, um Strategien entwickeln zu können, die das Teilen von Wissen zwischen verschiedenen Altersgruppen fördern. Auch Gemeinsamkeiten zu erkennen ist wichtig, da diese Unternehmen Einblicke in Bereiche geben, die sie weiter unterstützen sollten.

\section{Methodisches Vorgehen: Quantitative Umfrage und zugrundeliegendes Forschungsmodell}

Die Erforschung von Unterschieden und Gemeinsamkeiten zwischen jüngeren und älteren Arbeitnehmern deutscher Unternehmen erfolgte anhand einer quantitativen Methode. Hierzu wurde ein Forschungsmodell (Abb. 1) entwickelt, das verschiedene Konzepte aus der Literatur kombiniert und mit einer Online-Umfrage überprüft wurde. Dieses zugrundeliegende Modell basiert auf Theorien, die sowohl aus der Forschung zu Generationen als auch aus dem Wissensmanagement herangezogen wurden.

Verschiedene Beziehungen von Konstrukten sollen somit durch das bereits bestätigte Zusammenwirken verschiedener Faktoren erklärt werden. Im Entwicklungsprozess dient die „Theory of Reasoned Action“ von Fishbein und Ajzen (1975) und Ajzen und Fishbein (1980) als Ausgangspunkt. Mit Hilfe dieser Theorie wird versucht, bewusstes, menschliches Verhalten und dessen Intention durch die Einstellung und subjektive Norm des Umfelds von Individuen zu erklären. Diese wird durch die „Social
Exchange Theory“ von Blau (1964) erweitert. Nach dieser Theorie wägen Menschen vor einer Aktion ihren erwarteten Nutzen und ihre wahrgenommenen Kosten ab. In dem Modell stellt somit der Vorteil eine gewisse Motivation dar und Kosten repräsentieren empfundene Hürden der Teilnehmer.

Nach der "Social Identity Theory“ von Tafjel und Turner (1986) unterscheidet sich die Wahrnehmung von Individuen je nachdem, ob sie sich einer Gruppe zugehörig oder fremd fühlen. Eine Interaktion mit Personen mit vermeintlich ähnlichen oder gleichen Eigenschaften wird als angenehmer wahrgenommen und bevorzugt (Burmeister, Van der Heijden, Yang \& Deller, 2018, S. 607). In Bezug auf das Kollegium werden die Gruppen in jüngere und ältere Mitarbeiter mit unterschiedlicher Arbeitserfahrung und Dauer im Unternehmen eingeteilt.

Nach der „Espoused National Culture“-Theorie unterscheiden sich nicht nur Populationen hinsichtlich ihres kulturellen Empfindens, sondern auch Individuen innerhalb von Kulturkreisen (Hofstede, 2001; Srite \& Karahanna, 2006, S. 681). Die Gesellschaft in Deutschland wird sowohl altersmäßig als auch kulturell diverser, weshalb auch hier Unterschiede zu vermuten sind.

Der erstellte Fragebogen ${ }^{2}$ basiert auf dem in Abbildung 1 dargestellten Forschungsmodell und besteht aus insgesamt 13 Konstrukten. Konstrukte sind Konzepte, die sich durch Teilfragen (Items) messen lassen und nicht mit ja oder nein beantwortet werden können. Diese wurden aus der bestehenden Forschung abgeleitet und mit Items bereits durchgeführter und valider Umfragen angereichert. Die Items der Konstrukte wurden für den Kontext modifiziert und von den Teilnehmern auf einer 7-PunktLikert-Skala (1: Stimme voll und ganz zu; 7: Stimme überhaupt nicht zu) beantwortet.

Der Fragebogen beinhaltet folgende Konstrukte: Intention, Einstellung, subjektive Norm, Motivation (Vertrauen, Teamarbeit, Zugehörigkeit/Verbundenheit mit dem Unternehmen, Selbstwirksamkeit, Gegenseitigkeit, extrinsische Anreize), Hürden (Angst, Macht/Stellung zu verlieren, Gesichtsverlust), Individualismus vs. Kollektivismus und Stereotype gegenüber jüngeren Arbeitnehmern. So besteht zum Beispiel das Konstrukt subjektive Norm aus zwei Teilfragen (Items): „Ich denke, dass Kollegen, die wichtig für mich sind, erwarten, dass ich mein Wissen teile“, „Ich denke, dass Kollegen, deren Meinung ich schätze, erwarten, dass ich mein Wissen teile“. Tabelle 1 stellt den Ablauf und Inhalt des Fragebogens auf konzeptioneller Ebene dar.

2 Bei Interesse an dem Fragebogen nehmen Sie bitte Kontakt auf. 


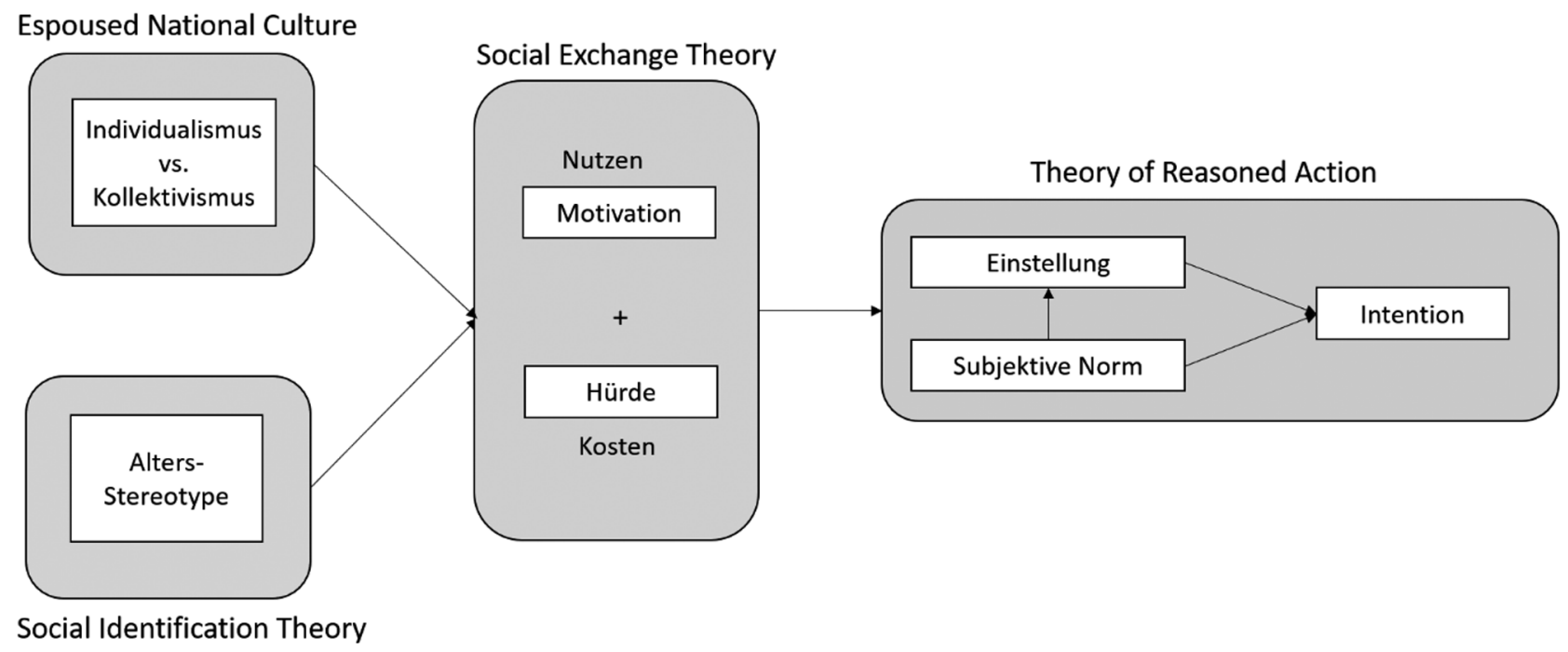

Abbildung 1: Modellstruktur nach verschiedenen Theorien (Blau, 1964; Fishbein \& Ajzen, 1975; Ajzen \& Flshbein, 1980; Tafjel, Turner, 1986; Hofstede, 2001; Srite \& Karahanna, 2006).

Tabelle 1: Fragebogenstruktur.

\begin{tabular}{ll}
\hline Bearbeitungsabschnitt & Inhalt \\
\hline Einleitung & - Begrüßung \\
& - Erreichen der Zielgruppe (Arbeitnehmer \\
& deutscher Unternehmen) \\
& - Motivation und Grund der Befragung \\
\hline Einstiegsfrage & Einleitung in das Szenario: \\
& „Haben Sie jemals Wissen mit ihren \\
& Kollegen geteilt?“ \\
\hline Wissensteilung & Hauptteil der Umfrage: \\
& - 13 Faktoren (z. B. Vertrauen, \\
& Teamarbeit) mit 32 Teilfragen \\
& - Zufällige Reihung der Konstrukte und \\
& Items \\
& - Darstellung des Konstruktes auf \\
& einzelnen Seiten \\
\hline Demographische Daten & Alter, Geschlecht, Land, Bildungsgrad, \\
& Arbeitserfahrung, Berufsjahre im \\
& Unternehmen, Typ und Größe des \\
& Unternehmens, Führungstätigkeiten \\
\hline
\end{tabular}

Die Auswertung des Fragebogens erfolgte in Form eines Strukturgleichungsmodells mit der Software PLS (Partial Least Squares) (Ringle, Wende \& Will, 2005). Hierbei wurden verschiedene Werte berechnet: Cross Loading, Cronbachs Alpha und Average Variance Extracted (AVE).

Alle Item-Werte mit mehr als 0,4 im Cross Loading sind repräsentativ für die Gültigkeit der Messwerte (Fornell \& Lacker, 1981; Hair, Tatham, Anderson \& Black, 1998). Cronbachs Alpha steht für die Zuverlässigkeit der Konsistenz der Konstrukte mit den dazugehörigen Skalen und Messitems. Hierbei wird getestet, ob die einzelnen
Items das entsprechende Konstrukt messen. Der Wert muss mindestens 0,6 erreichen (Cronbach, 1984; Nunually, 1978; Hair, Black, Babin, Anderson \& Tatham, 2006). Die konvergente Zuverlässigkeit des Konstrukts wird durch den AVE-Wert bestimmt, der einen Mindestwert von 0,5 erreichen muss (Hair et al., 2006). Alle verwendeten Items und Konstrukte weisen die Mindestwerte auf und können somit für die Auswertung herangezogen werden.

Die Rekrutierung der Teilnehmer erfolgte durch das Anschreiben verschiedener Unternehmen, Geschäftsführer und Arbeitnehmer aus dem Bekanntenkreis und dem Teilen des Umfragelinks auf sozialen Netzwerken mit der Bitte zur Weiterleitung. Der Erhebungszeitraum erstreckte sich vom 07.05.2019 bis zum 01.06.2019.

\section{Ergebnisse}

Insgesamt beantworteten 236 Arbeitnehmer aus deutschen Organisationen den Online-Fragebogen vollständig. Diese stammen aus unterschiedlichen Branchen und variieren im Organisationstyp und in der Anzahl der Beschäftigten.

In der vorliegenden Untersuchung werden zwei Generationen unterschieden: Jüngere und ältere Arbeitnehmer. Das primäre Unterscheidungskriterium ist das Alter der Personen, welches signifikant positiv mit der Berufserfahrung und den Berufsjahren im Unternehmen korreliert (siehe Tabelle 2). Durch das Heranziehen mehrerer T-Tests wurde untersucht, wann die beiden Gruppen in sich möglichst homogen und im Vergleich möglichst heterogen in 
den verschiedenen Konstrukten sind. Der entscheidende Wendepunkt liegt in diesem Teilnehmer-Pool bei 39 Jahren.

Tabelle 2: Korrelationen.

\begin{tabular}{lllll}
\hline & & Alter & Erfahrung & Dauer \\
\hline Alter & Pearson Correlation & 1 & & \\
& Sig. (2-geteilt) & 236 & & \\
& $\mathrm{~N}$ & & & \\
\hline \multirow{2}{*}{ Erfahrung } & Pearson Correlation & $.830^{\star \star}$ & 1 & \\
& Sig. (2-geteilt) & .000 & 236 & \\
& $\mathrm{~N}$ & 236 & & \\
\hline \multirow{2}{*}{ Dauer } & Pearson Correlation & $.671^{\star \star}$ & $.732^{\star \star}$ & 1 \\
& Sig. (2-geteilt) & .000 & .000 & 236 \\
& $\mathrm{~N}$ & 236 & 236 & \\
\hline
\end{tabular}

${ }^{\star \star}$. Korrelation ist signifikant ab einem Level von 0.01 (zweigeteilt)
Nach dieser Gruppeneinteilung sind es 94 ältere Teilnehmer von 39 bis 64 Jahren mit einem Durchschnittsalter von 51 Jahren, der durchschnittlichen Dauer im Unternehmen von 10 bis 15 Jahren und einer durchschnittlichen Berufserfahrung von mehr als 15 Jahren. Die jüngeren Arbeitnehmer sind 18 bis 38 Jahre alt, mit einem Durchschnittsalter von 28 Jahren, der durchschnittlichen Dauer von ein bis zwei Jahren im Unternehmen und einer durchschnittlichen Berufserfahrung von drei bis fünf Jahren. Die demographische Verteilung der Personen in Bezug auf Unternehmensgröße, Unternehmensart, Abschluss und weitere demographische Aspekte können als Prozentangaben der Tabelle 3 entnommen werden.

Tabelle 4 gibt einen Überblick der Aufteilung, welche Faktoren welche Gruppe beeinflussen. Auf diese wird im Folgenden detaillierter eingegangen.

Tabelle 3: Übersicht demographischer Daten.

\begin{tabular}{|c|c|c|c|c|}
\hline Demographische Variable & Kategorie & Alle Teilnehmer & Jüngere Teilnehmer & Ältere Teilnehmer \\
\hline & & $\%$ & $\%$ & $\%$ \\
\hline \multirow[t]{3}{*}{ Geschlecht } & Männlich & 54,2 & 52,1 & 57,4 \\
\hline & Weiblich & 41,5 & 45,8 & 35,1 \\
\hline & Keine Antwort & 4,2 & 2,1 & 7,4 \\
\hline \multirow[t]{2}{*}{ Herkunftsland } & Deutschland & 94,5 & 95,8 & 92,6 \\
\hline & Anderes Land & 5,5 & 4,2 & 7,4 \\
\hline \multirow[t]{5}{*}{ Höchster Abschluss } & Qualifizierter Hauptschulabschluss & 3,4 & 2,1 & 5,3 \\
\hline & Mittlere Reife & 19,1 & 14,8 & 25,5 \\
\hline & Abitur & 10,6 & 9,9 & 11,7 \\
\hline & Hochschulabschluss & 59,3 & 65,5 & 50,0 \\
\hline & Sonstiges & 7,6 & 7,7 & 7,4 \\
\hline \multirow[t]{5}{*}{ Unternehmensart } & Öffentliche Einrichtung & 4,7 & 4,2 & 5,3 \\
\hline & Öffentliches Unternehmen (z. B. Stadtwerke) & 6,8 & 10,6 & 1,1 \\
\hline & Privatunternehmen/ Konzern & 51,3 & 60,6 & 37,2 \\
\hline & Familienunternehmen & 32,6 & 19,7 & 52,1 \\
\hline & Sonstiges & 4,7 & 4,9 & 4,3 \\
\hline \multirow[t]{10}{*}{ Unternehmenssektor } & Energie & 2,5 & 2,8 & 2,1 \\
\hline & Materialien & 2,1 & 1,4 & 3,2 \\
\hline & Industrie & 24,6 & 14,8 & 39,4 \\
\hline & Gebrauchsgüter & 2,5 & 4,2 & 0,0 \\
\hline & Verbrauchsgüter & 3,0 & 3,5 & 2,1 \\
\hline & Gesundheitswesen & 6,8 & 8,5 & 4,3 \\
\hline & Finanzkennzahlen & 1,7 & 2,8 & 0,0 \\
\hline & Informationstechnologie & 6,4 & 10,6 & 0,0 \\
\hline & Kommunikationsdienste & 2,1 & 2,1 & 2,1 \\
\hline & Versorgungsunternehmen & 1,7 & 2,1 & 1,1 \\
\hline
\end{tabular}




\begin{tabular}{|c|c|c|c|c|}
\hline Demographische Variable & Kategorie & Alle Teilnehmer & Jüngere Teilnehmer & Ältere Teilnehmer \\
\hline & & $\%$ & $\%$ & $\%$ \\
\hline & Immobilien & 5,1 & 5,6 & 4,3 \\
\hline & Dienstleister & 26,7 & 26,1 & 27,7 \\
\hline & Sonstiges & 14,8 & 15,5 & 13,8 \\
\hline \multirow[t]{6}{*}{ Beschäftigte } & $<50$ & 23,7 & 29,6 & 14,9 \\
\hline & $50-100$ & 5,9 & 9,2 & 1,1 \\
\hline & $100-200$ & 21,6 & 8,5 & 41,5 \\
\hline & $200-500$ & 20,8 & 13,4 & 31,9 \\
\hline & $500-1000$ & 3,0 & 4,2 & 1,1 \\
\hline & $>1000$ & 25,0 & 35,2 & 9,6 \\
\hline \multirow[t]{6}{*}{ Dauer im Unternehmen } & $<1$ & 17,4 & 25,4 & 5,3 \\
\hline & $1-2$ & 25,4 & 35,9 & 9,6 \\
\hline & $3-5$ & 19,9 & 24,6 & 12,8 \\
\hline & $6-9$ & 8,1 & 9,2 & 6,4 \\
\hline & $10-15$ & 10,2 & 2,8 & 21,3 \\
\hline & $>15$ & 19,1 & 2,1 & 44,7 \\
\hline \multirow[t]{6}{*}{ Berufserfahrung } & $<1$ & 4,7 & 7,7 & 0 \\
\hline & $1-2$ & 16,1 & 26,8 & 0 \\
\hline & $3-5$ & 19,5 & 32,4 & 0 \\
\hline & $6-9$ & 11,4 & 17,6 & 2,1 \\
\hline & $10-15$ & 11,0 & 11,3 & 10,6 \\
\hline & $>15$ & 37,3 & 4,2 & 87,2 \\
\hline \multirow[t]{2}{*}{ Führungstätigkeit } & $\mathrm{Ja}$ & 33,9 & 27,5 & 43,6 \\
\hline & Nein & 66,1 & 72,5 & 56,4 \\
\hline
\end{tabular}

Tabelle 4: Übersicht der Ergebnisse.

\begin{tabular}{llll}
\hline & Alle Teilnehmer & Ältere Teilnehmer & Jüngere Teilnehmer \\
\hline Motivation & - Einstellung Wissensteilung & - Subjektive Norm & Selbstwirksamkeit \\
& - Gegenseitigkeit & - Zugehörigkeit & \\
& - Vertrauen & - Gewichtung Vertrauen & \\
& - Teamarbeit & - Gewichtung Teamarbeit & \\
\hline Hürden & Verlust der Stellung & & Gesichtsverlust \\
\hline Hoher Kollektivismus & - Verbundenheit & Teamarbeit & \\
& - Gegenseitigkeit & & \\
& - Extrinsische Anreize & & \\
\hline Altersstereotype & - Gesichtsverlust & & Vertrauen \\
& - Einstellung & Gesichtsverlust & \\
\hline
\end{tabular}

\section{Motivation}

Es ist für alle Teilnehmer wichtig, dass das Teilen von Wissen mit Kollegen als angenehm, gut, wertvoll und nützlich empfunden wird. Dieses wird positiv durch die Faktoren der Gegenseitigkeit, des Vertrauens und der Teamarbeit beeinflusst.
Das Gefühl, Gegenseitigkeit zu empfinden, ist für alle ein motivierender Einflussfaktor. Das Teilen von Wissen soll die Beziehung stärken, den Umfang der Zusammenarbeit erweitern und das Teilen von Wissen der anderen Person gewährleisten.

Auch Vertrauen gegenüber den anderen Personen im Arbeitsumfeld spielt eine große Rolle. Die Teilnehmer 
wünschen sich, ihre Ideen, Gefühle und Hoffnungen mit ihren Kollegen zu teilen und offen über Probleme reden zu können, wobei eine einfühlsame Reaktion erwartet wird.

In Bezug auf Teamarbeit teilen Beschäftigte ihr Wissen lieber, wenn die Zusammenarbeit mit den Kollegen kooperativ wahrgenommen wird, diese gute Zuhörer bei Problemen sind und Meinungsverschiedenheiten gemeinsam gelöst werden. Das Funktionieren als Team ist ebenfalls essentiell. Obwohl es allen Teilnehmern wichtig ist, hohes Vertrauen und eine gute Teamarbeit mit den Kollegen zu pflegen, empfinden ältere Arbeitnehmer diese Faktoren doppelt so wichtig als ihre jüngeren Kollegen.

Die wahrgenommene Erwartungshaltung von Personen (subjektive Norm), Wissen zu teilen, impliziert den Einfluss und den wahrgenommenen sozialen Druck von besonders geschätzten und wichtigen Kollegen am Arbeitsplatz. Die Meinung und der Einfluss dieser Personen ist den Betroffenen viel Wert. Bei jüngeren Personen hat die Erwartung des Kollegiums im Gegensatz zu älteren Mitarbeitern keinerlei Auswirkung auf die Intention, Wissen zu kommunizieren.

Ältere Personen empfinden das Teilen angenehmer, wenn sie eine hohe Zugehörigkeit zum Unternehmen verspüren, verbunden mit der Freude, den Rest der Karriere in der aktuellen Firma zu verbringen. Auch das Zugehörigkeitsgefühl zum Arbeitsort ist von Bedeutung.

Den jüngeren Teilnehmern hingegen ist die eigene Selbstwirksamkeit sehr wichtig. Hierbei sind sie in der Lage, dem Unternehmen nützliches Fachwissen bereitzustellen und den Kollegen einen Mehrwert zu liefern.

Extrinsische Anreize wie Gehaltserhöhungen, Boni, eine höhere Jobsicherheit oder eine bessere Förderung als Gegenleistung sind als motivierende Faktoren nicht signifikant.

\section{Hürde}

In Bezug auf Hürden befürchten alle Teilnehmer den Verlust ihrer Stellung. Im Äußern ihres Wissens empfinden sie demnach das Gefühl, weniger Macht zu haben und ihren einzigartigen Wert in der Organisation zu verlieren. Nur die jüngere Generation verspürt die Angst, Fehler zu begehen oder sich lächerlich zu machen, wenn sie ihr Wissen mit Kollegen teilt. Diese Furcht wird Gesichtsverlust (engl. Loosing Face) genannt.

\section{Beeinflussende Faktoren}

Altersstereotype beinhalten wahrgenommene und angenommene Unterschiede in Fähigkeiten und Verhaltensmustern von Personen unterschiedlichen Alters. Diese wurden in der Umfrage nur in Bezug auf jüngere Personen erhoben. Trotz dieser einseitigen Erhebung lassen sich negative Auswirkungen bei allen Teilnehmern erkennen. Sobald im Vergleich jüngere Arbeitnehmer als unproduktiv und unflexibel wahrgenommen werden, empfinden alle Teilnehmer das Teilen von Wissen als weniger angenehm, gut, wertvoll und nützlich. Auch die Qualität der Teamarbeit sinkt. Jüngere Mitarbeiter verlieren zudem an Vertrauen gegenüber ihren Kollegen, ältere Personen werden in ihrer Angst, sich zu blamieren, bestärkt.

In Bezug auf ein hohes kollektives Empfinden verspüren alle Teilnehmer unabhängig von ihrer Gruppenzugehörigkeit eine gesteigerte Verbundenheit mit dem Unternehmen, gestärkte Gegenseitigkeit, aber auch mehr Gefallen an extrinsischen Anreizen beim Teilen von Wissen. Nur bei älteren Personen verbessert sich die Wahrnehmung von guter Teamarbeit, umso kollektivistischer sie eingestellt sind. Beide Generationen haben jedoch mehr Angst, Fehler zu begehen und sich lächerlich zu machen. Kollektivistische Personen empfinden in diesem Kontext Gruppenakzeptanz wichtiger als Autonomie und Unabhängigkeit, Gruppenerfolg wichtiger als individuellen Erfolg und Loyalität wichtiger als individuellen Gewinn (Hofstede, 2001; Srite \& Karahanna, 2006).

\section{Diskussion und Auswirkungen für Unternehmen}

Die Ergebnisse lassen bereits Schlüsse auf die Intention des Teilens von Wissen in den verschiedenen Altersgruppen zu. Viele getestete Konstrukte wie Vertrauen, Teamarbeit oder die Angst, durch das Teilen von Wissen Macht zu verlieren, stimmen mit den in der Literatur beschriebenen Befunden überein. Einige Faktoren betreffen beide Gruppen gleichermaßen, aber es zeigen sich auch Unterschiede.

Unternehmen sollten deshalb unterschiedliche Bereiche betrachten, wenn sie alle oder explizit jüngere oder ältere Personen ansprechen möchten. Zur Stärkung der Wissensteilung aller Mitarbeiter liegt die Empfehlung nahe, vor allem darauf $\mathrm{zu}$ achten, dass vertrauensvolle Beziehungen gepflegt werden. Kooperative Teamarbeit sollte gefördert und gegenseitige Motivation unterstützt werden. 
Insbesondere für die jüngeren Arbeitnehmer ist es wichtig, dass das Selbstvertrauen gestärkt wird. Den betreffenden Personen sollte die Angst genommen werden, sich lächerlich zu machen oder Fehler zu begehen, wenn sie ihr Wissen teilen. Hier könnte es sinnvoll sein, die Fehlerkultur im Unternehmen zu untersuchen und zu modifizieren.

Durch eine intensivere Bindung älterer Personen an das Unternehmen kann der freiwillige Prozess der Wissensteilung unterstützt werden. Hierzu ist es wichtig zu verstehen, welche Faktoren für die Mitarbeiter ausschlaggebend für ein höheres Zugehörigkeitsgefühl sind und einen langfristigen Verbleib im Unternehmen vorstellbar machen.

Extrinsische Anreize wie Boni, Gehaltserhöhungen oder außergewöhnliche Förderung zeigen bei keiner Gruppe einen Effekt in der Intention, Wissen zu teilen. Demnach kann dieser Aspekt bei der Strategieentwicklung im Bereich Wissensmanagement vernachlässigt werden.

Ein persönlicher Mehrwert und ein gutes Unternehmensklima erweisen sich als ausschlaggebend. Folglich müssen altersgruppenbasierte Strategien für die Beschäftigten generiert werden, die sich zum Beispiel von der Personalabteilung umsetzen lassen. Hierzu könnte die Definition von Wissen im jeweiligen Unternehmen zählen sowie das Kommunizieren, dass sich ein Wettbewerbsvorteil nicht durch das Zurückhalten von Wissen erlangen lässt. Ziel sollte es sein, Wissen als Allgemein- und Unternehmensgut zu sehen. Dies ist wichtig, um Wissenslücken zu vermeiden, gemeinsam weiterzukommen und erfolgreich zu sein.

Für ein positives Arbeitsklima ist auch das Vermeiden von Altersstereotypen wesentlich. Diese wirken sich negativ auf wichtige Einflussfaktoren der Arbeitnehmer aus, ihr Wissen $\mathrm{zu}$ teilen. Hierbei ist es unwichtig, welcher Gruppe die betreffenden Personen zugehörig sind. Das reine Vorhandensein von Altersstereotypen bringt negative Konsequenzen an den Arbeitsplatz. Der Austausch unter den Generationen muss gefördert werden, indem unter anderem auch Verständnis geschaffen wird. Hier könnten verschiedene Strategien herangezogen werden, um einen positiven und direkten Austausch zu fördern. Sowohl ältere als auch jüngere Beschäftigte haben Kompetenzen in verschiedenen Bereichen, die für die jeweils andere Gruppe einen Mehrwert bringen können.

\section{Fazit und Ausblick}

Generationen am Arbeitsplatz unterscheiden sich nicht nur durch die Einteilung in Baby-Boomer und Generation X, Y, Z. Auch das biologische Alter ist kein geeignetes Unterscheidungsmerkmal. Weitere Charakteristika im Unternehmen müssen beachtet werden. In diesem Beitrag handelt es sich dabei um die Berufserfahrung und die Betriebszugehörigkeit. Davon abgeleitet werden die Personen in jüngere $(\mathrm{N}=142)$ und ältere $(\mathrm{N}=94)$ Personen eingeteilt, weil sich auch der Wissensstand und die Art des Wissens im Laufe der Zeit verändern.

Die 236 Teilnehmer deutscher Unternehmen gruppieren sich in jüngere Arbeitnehmer zwischen 18 und 38 Jahren und ältere zwischen 39 und 64 Jahren. Aus der OnlineUmfrage ist zu erkennen, dass jüngere Personen größere Hürden verspüren, ihr Wissen zu teilen. Sie haben Angst, sich zu blamieren oder falsches Wissen zu verbreiten. Selbst die Erwartungshaltung ihrer Kollegen hat keinen Einfluss auf die Intention des Teilens. Ältere Personen hingegen werden durch die Erwartung anderer Personen angeregt, ihr Wissen zu kommunizieren.

Für alle Teilnehmer ergibt die durchgeführte Studie, dass extrinsische Anreize vernachlässigbar sind. Sie haben keine Auswirkung auf das Teilen von Wissen. Einige Faktoren hingegen beeinflussen beide Gruppen, andere nur die jüngeren oder älteren Arbeitnehmer. Die Betrachtung der einzelnen Faktoren ist essentiell, um den Austausch zu fördern, Wissenslücken zu vermeiden und ein Lernen voneinander zu ermöglichen. Hierbei wäre es unter anderem interessant zu erforschen, wie sich eine Unterscheidung zwischen implizitem und explizitem Wissen auf die Intention und das Teilen auswirkt. Der Schwierigkeitsgrad der Wissensarten muss hierbei betrachtet werden (Nonaka, 1994). Ein weiterer Ansatz setzt auf verschiedene Methoden und Technologien wie Social Tools, die den Wissensaustausch fördern und das Teilen erleichtern können. Hierbei sollte die Branche berücksichtigt werden, da diese unterschiedliche Wissensgemeinschaften, Persönlichkeiten, Fähigkeiten und Firmenkulturen bedingen kann.

In Bezug auf die Altersunterschiede und damit einhergehenden objektiven Stereotypen sollten Unternehmen genauer auf eine vorurteilsfreie Umgebung achten und die Auswirkungen auf Zusammenarbeit, Kommunikation und das Teilen von Wissen mehr in den Fokus rücken. 


\section{Literatur}

Ajzen, I. \& Fishbein, M. (1980). Understanding Attitudes and Predicting Social Behavior, Prentice Hall, Englewood Cliffs, N).

Anand, A., \& Singh, M. D. (2011). Understanding knowledge management. International Journal of Engineering Science and Technology, 3(2), 926-939.

Blau, P. M. (1964). Exchange and power in social life. NY: John Wiley \& Sons.

Bock, G.-W., Zmund, W. R., Kim, Y.-G., \& Lee, J.-N. (2005). Behavioral Intention Formation in Knowledge Sharing: Examining the Roles of Extrinsic Motivators, SocialPsychological Forces, and Organizational Climate. MIS Quarterly, 29(1), 87-111.

Burmeister, A., \& Deller, J. (2016). Knowledge Retention From Older and Retiring Workers: What Do We Know, and Where Do We Go From Here? Work, Aging and Retirement, 2(2), 87-104.

Burmeister, A., van der Heijden, B., Yang, J., \& Deller, J. (2018). Knowledge transfer in age-diverse coworker dyads in China and Germany: How and when do age-inclusive human resource practices have an effect? Human Resource Management Journal, 28(4), 605-620.

Cennamo, L., \& Gardner, D. (2008). Generational differences in work values, outcomes and person-organisation values fit. Journal of Managerial Psychology, 23(8), 891-906.

Costanza, D. P., Badger, J. M., Fraser, R. L., Severt, J. B., \& Gade, P. A. (2012). Generational Differences in Work-Related Attitudes: A Meta-analysis. Journal of Business and Psychology, 27(4), 375-394.

Cronbach, L. J. (1984). Essentials of psychological testing. New York: Harper and Row.

DeLong, D. W. (2004). Lost Knowledge: Confronting the Threat of an Aging Workforce, Oxford University Press, New York, NY.

Ellwart, T., Bündgens, S., \& Rack, O. (2013). Managing knowledge exchange and identification in age diverse teams. Journal of Managerial Psychology, 28(7/8), 950- 972.

Ely, R. J. (1994). The Effects of Organizational Demographics and Social Identity on Relationships among Professional Women. Administrative Science Quarterly, 39(2), 203-238.

Eurostat (2019). Population structure and ageing. Abgerufen von: https://ec.europa.eu/eurostat/statistics-explained/pdfscache/ 1271.pdf [22.5.2020]

Farooq, R. (2018). A conceptual model of knowledge sharing. International Journal of Innovation Science, 10(2), 238-260.

Fishbein, M. \& Ajzen, I. (1975). Belief, Attitude, Intention, and Behavior: An Introduction to Theory and Research, Addison-Wesley, Reading, MA.

Fornell, C., \& Larcker, D. F. (1981). Evaluating structural equations with unobservable variables and measurement error. Journal of Marketing Research, 18(1), 39-50.

Girard, J., \& Girard, J. (2015). Defining knowledge management: Toward an applied compendium. Online Journal of Applied Knowledge Management, 3(1), 1-20.

German Trade \& Invest (2020). Demographic Change. Global Megatrend. Abgerufen von: https://www.gtai.de/gtai-en/invest/in dustries/demographic-change-65744 [27.4.2020]

Hair J.F, Tatham R.L, Anderson R.E, \& Black W. C. (1998). Multivariate Data Analysis, 5th ed. Upper Saddle River, NJ: Prentice Hall.
Hair, J.F., Black, W. C., Babin, B. J., Anderson, R. E. \& Tatham, R. L. (2006). Multivariate data analysis. Prentice Hall Pearson Education.

Hippel, C. von, Kalokerinos, E. K., \& Henry, J. D. (2013). Stereotype threat among older employees: Relationship with job attitudes and turnover intentions. Psychology and Aging, 28(1), 17-27.

Hofstede, G. (2001). Culture's Consequences: Comparing Values, Behaviors, Institutions, and Organisations Across Nations, Sage Publication, London.

Iweins, C., Desmette, D., Yzerbyt, V., \& Stinglhamber, F. (2013). Ageism at work: The impact of intergenerational contact and organizational multi-age perspective. European Journal of Work and Organizational Psychology, 22(3), 331-346.

Joy, A., \& Haynes, B. P. (2011). Office design for the multi-generational knowledge workforce. Journal of Corporate Real Estate, 13 (4), 216-232.

Kankanhalli, A., \& Tan, B. C. Y. (2005). Contributing Knowledge to electronic knowledge repositories: An empirical investigation. MIS Quarterly, 29(1), 113-143.

Klaffke, M., \& Schwarzenbart, U. (2013). Demografie als Chance. Personalmagazin, 03, 44-47.

Krok, E. (2013). Willingness to Share Knowledge Compared with Selected Social Psychology Theories. Contemporary Economics, 7(1), 101-109.

Kunze, F., \& Menges, J. I. (2017). Younger supervisors, older subordinates: An organizational-level study of age differences, emotions, and performance. Journal of Organizational Behavior, 38 (4), 461-486.

Kunze, F., Boehm, S. A., \& Bruch, H. (2011). Age diversity, age discrimination climate and performance consequences-a cross organizational study. Journal of Organizational Behavior, 32(2), 264-290.

Kuyken, K. (2012). Knowledge communities: towards a re-thinking of intergenerational knowledge transfer. VINE, 42(3/4), 365-381.

Lagacé, M., Charmarkeh, H., Zaky, R., \& Firzly, N. (2016). From psychological to digital disengagement: exploring the link between ageism and the 'grey digital divide.' Romanian Journal of Communication and Public Relations, 18(1), 65-75.

Lyons, S. T., \& Schweitzer, L. (2016). A Qualitative Exploration of Generational Identity: Making Sense of Young and Old in the Context of Today's Workplace. Work, Aging and Retirement, 3 (2), 209-224.

McGuire, D., Todnem By, R., \& Hutchings, K. (2007). Towards a model of human resource solutions for achieving intergenerational interaction in organisations. Journal of European Industrial Training, 31(8), 592-608.

Nonaka, I. (1994). A Dynamic Theory of Organizational Knowledge Creation. Organisational Science, 5(1), 14-37.

Nunually J. (1978). Psychometric Theory. New York: McGraw-Hill.

Pee, L. G., \& Lee, J. (2015). Intrinsically Motivating Employees' Online Knowledge Sharing: Understanding the Effects of Job Design. International Journal of Information Management, 35(6), 679690.

Peters, P., Van der Heijden, B. I. J. M., Spurk, D., De Vos, A., \& Klaassen, R. (2019). Please Don't Look at Me That Way. An Empirical Study Into the Effects of Age-Based (Meta)-Stereotyping on Employability Enhancement Among Older Supermarket Workers. Frontiers in Psychology, 10 (February), 1-14. 
Rahman, M. S., Daud, N. M., \& Hassan, H. (2017). Generation "X" and "Y" knowledge sharing behaviour. Journal of Applied Research in Higher Education, 9(2), 325-342.

Razak, N. A., Pangil, F., Zin, M. L. M., Yunus, N. A. M., \& Asnawi, N. H. (2016). Theories of Knowledge Sharing Behavior in Business Strategy. Procedia Economics and Finance, 37(16), 545-553.

Reychav, I., \& Weisberg, J. (2010). Bridging intention and behavior of knowledge sharing. Journal of Knowledge Management, 14(2), 285-300.

Ringle, C., Wende, S. \& Will, A. (2005). SmartPLS 2.0. M3. Hamburg: SmartPLS. Abgerufen von: http://www.smartpls.com [Letzter Zugriff: 27-04-2020]

Rudolph, C. W., \& Zacher, H. (2015). Intergenerational perceptions and conflicts in multi-age and multigenerational work environments. In Fraccaroli, F., Truxillo, D. M., Finkelstein, L. M., \& Kanfer, R., (Eds.), Facing the Challenges of a Multi-Age Workforce: A Uselnspired Approach, (pp.253-282). New York: Routlege.

Slagter, F. (2007). Knowledge management among the older workforce. Journal of Knowledge Management, 11(4), 82-96.

Srite, M., \& Karahanna, E. (2006). The Role of Espoused National Cultural Values in Technology Acceptance. MIS Quarterly, 30(3), 679-704.

Tajfel, H., \& Turner, J. C. (1986). The social identity theory of intergroup behavior. In Worchel S., \& Austin, W. G. (Eds.), Psychology of intergroup relation, (pp.7-24). Chicago: Nelson Hall.

Widjaja, M.E. L. (2019). Determinants of knowledge sharing studies: organizational contexts in the social perspective. In Proceedings of the 16th International Symposium on Management (INSYMA 2019) (Vol. 87-90). Paris, France: Atlantis Press.

Wok, S., \& Hashim, J. (2013). Communicating and sharing working relationships with older employees. Journal of Communication Management, 17(2), 100-121.

Woodfield, P., \& Husted, K. (2017). Intergenerational knowledge sharing in family firms: Case-based evidence from the New Zealand wine industry. Journal of Family Business Strategy, 8(1), 57-69.

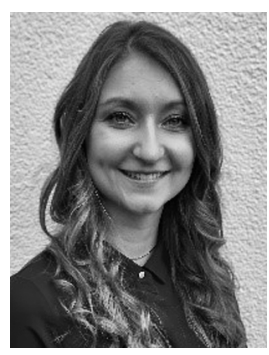

\section{Sophie März}

Universität Hildesheim

Institut für Informationswissenschaft und

Sprachtechnologie

Universitätsplatz 1

31141 Hildesheim

maerz@uni-hildesheim.de

Sophie März ist wissenschaftliche Mitarbeiterin und Doktorandin an der Universität Hildesheim. Sie hat ihren Bachelor „Internationales Informationsmanagement" in Hildesheim und ihren Masterabschluss 2019 in einem Joint Degree zusammen mit der Chungbuk National University in Südkorea absolviert.

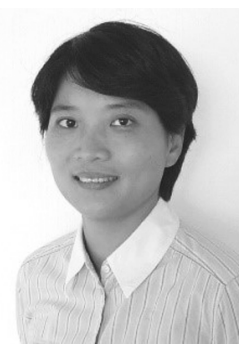

Prof. DongBack Seo, Ph. D.

Chungbuk National University

Department of Management Information

Systems

567 Baekje-daero

Deokijn-gu

Jeonju-si, Jeollabuk-do

54896 Republic of Korea

dseo@chungbuk.ac.kr

Prof. DongBack Seo promovierte an der University of Illinois in Chicago und erwarb dort einen Master of Science in Management Information Systems. Davor arbeitete sie als Softwareingenieurin in einem Unternehmen für drahtlose Kommunikation und als Inhaberin eines Kleinunternehmens. Sie hat bereits drei Bücher und zahlreiche Zeitschriftenartikel veröffentlicht. Ihr Forschungsinteresse gilt der digitalen Innovation, der Einführung digital unterstützter Dienste aus der Sicht von Einzelpersonen und Organisationen, der Strategie organisatorischer Standards, der Konvergenz von Unternehmen und der Analyse der Wettbewerbsdynamik in sich schnell verändernden Branchen. Gegenwärtig ist sie Lehrstuhlinhaberin in der Abteilung für Management-Informationssysteme an der Chungbuk National University in Südkorea.

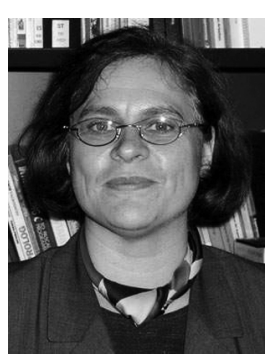

Prof. Dr. Christa Womser-Hacker

Universität Hildesheim

Institut für Informationswissenschaft und

Sprachtechnologie

Universitätsplatz 1

31141 Hildesheim

womser@uni-hildesheim.de

Prof. Dr. Christa Womser-Hacker ist Professorin für Informationswissenschaft an der Universität Hildesheim. Wissenschaftliche Arbeitsschwerpunkte stellen die Themenfelder Multilingualität und Interkulturalität in Informationssystemen, Evaluierung von InformationRetrieval-Systemen und Usability/User Experience - Mensch-Maschine-Interaktion dar. 